Cooke and Ross-Zeiss lenses. It may perhaps, therefore, be inferred from this, that it is at present only possible to photograph meteors which are moving slowly, or are un. usually bright. A second, much brighter and longer, meteor

Harvard College Observatory, I 904 Nov. 26. trail was photographed early in the evening. The positions, for 1855 , of its two ends are RA. $=4^{\mathrm{h}} 5^{2^{\mathrm{m}}} \cdot 5$, Decl. $=+0^{\circ} 5^{\circ}$, and RA. $=5^{\mathrm{h}} \mathrm{r}^{\mathrm{m}} \cdot 7$, Decl. $=-4^{\circ} 39^{\circ}$. It was therefore not a Leonid.

\section{Edward C. Pickering.}

\title{
Note on the Leonid meteors of 1904.
}

The weather was very fine in England at the epoch of the Leonids but unfortunately fogs were very prevalent at night.

On Nov. 14 the sky was pretty clear at Bristol and between $13^{\mathrm{h}} 30^{\mathrm{m}}$ and $15^{\mathrm{h}} 45^{\mathrm{m}}$, in a watch extending over about $1 \frac{1}{2}$ hour, 55 meteors were seen including 33 Leonids with a radiant at $15 \mathrm{I}^{\circ}+23^{\circ}$. I estimated that the Leonids were appearing at the rate of about 25 per hour for one observer, but the number exhibited no increase towards $16^{\text {h }}$ after which the fog became denser and observations were discontinued. Two Leonids were seen which were as bright

Bishopston, Bristol, I 904 Nov. I 7. as Jupiter and several others were as bright as, or brighter than, first magnitude stars. At $14^{\mathrm{h}} 3^{8^{\mathrm{m}}}$ there was a flash from a bright Leonid, in the $\mathrm{NW}$ sky, with a path from $315^{\circ}+57^{\circ}$ to $318^{\circ}+50^{1} /^{\circ}$ at the end of which it left a short streak for about 30 seconds.

The minor showers of the mid-November period appeared to be in stronger evidence than usual. I observed a few slow, yellow meteors from a radiant in Aries at $43^{\circ}+2 I^{\circ}$ and some swift, streaking meteors indicated a radiant in Leo minor at $144^{\circ}+37^{\circ}$.

\section{Uber den Stern BD. $+30^{\circ} 583$.}

Von Prof. E. Millosevich, Direktor der Sternwarte des Collegio Romano in Rom, ging am 14. Oktober folgende Mitteilung ein:

,Non so se sia stato notato che aumentando di $\mathrm{I}^{\mathrm{m}}$ $\alpha$ di BD. $+30^{\circ}: 58$, che è noto mancare in cielo, si cade in BD. $+30: 585$, percui l' errore di BD. sarebbe manifesto. «

Auf meine Bitte, die Originale der BD. für den Stern zu kontrollieren, schreibt Prof. $F$. Küistner am I9. Oktobef:

Der Ort des Sterns BD. $+30: 583$

$$
9.5 \quad 3^{\mathrm{h}} 43^{\mathrm{m}} \times 2^{\mathrm{s}} .7+30^{\circ} 4: 6
$$

beruhte auf folgenden Beobachtungen:

SZ. 820 Sch. I 856 Febr. 3 , sehr klare und durchsichtige Luft $9.5 \quad 3^{\mathrm{h}} 43^{\mathrm{m}} 9^{\mathrm{s}}: 8+30^{\circ} 4: 8$ alles deutlich und richtig, insbesondere die Zeitminute;

SZ. 785 Sch. 1855 Dez. 31 , wechselnde und zu dieser Zeit neblige Luft

$$
9.5 \quad 3^{\mathrm{h}} 44^{\mathrm{m}} 15^{\mathrm{s}} \cdot 7+30^{\circ} 4^{\prime} \cdot 5
$$

die Zeitminute heißt ganz zweifellos 44 ; sie ist fälschlich 43 gelesen und der Ort im Spezialkatalog $z u+30^{\circ} 5^{83}$ geschrieben, während er $\mathrm{zu}+300_{5} 85$ gehört.

Für den Stern $+30^{\circ} 5^{8} 3$ liegt also nur eine einzige Sucherbeobachtung vor und er ist schon aus diesem Grunde zu streichen; mit um so größerem Recht aber, wenn er jetzt am Himmel vermißt wird.

$\mathrm{Bei}+30: 585$ ändert sich durch das Hinzukommen der obigen Beobachtung die AR. rechnungsmäßig in $14^{3} \cdot 5$, die Dekl. bleibt ungeändert.

$K r$.

\section{Mitteilung aus den Originalen der BD.}

Über den Ort des Veränderlichen 188.1904 Draconis

$$
18^{\mathrm{h}} 40^{\mathrm{m}} 3^{2^{\mathrm{s}}}+62^{\circ} 3^{1}: \mathrm{I} \quad(\mathrm{r} 855)
$$

sind in der BD. gegangen die Sucherzonen (Teilwert 9:82):

Z. ${ }^{1367}$ Kr. 1857 Okt. 13, Luft sehr klar

an der Stelle ist nichts beobachtet, in der Nähe:

$9^{\mathrm{m}} \cdot 5 \quad 18^{\mathrm{h}} 40^{\mathrm{m}} 19^{\mathrm{s}}+62^{\circ} 20: 9$.

Z. I 378 Kr. 1857 Okt. 15 , Luft neblig und das Okular stets angelaufen

an der Stelle ist nichts beobachtet.

Z. I6 2 Sch. 1858 Juni 8 , klare Luft, Dämm. bricht an dicht an der Stelle ist beobachtet:

Sternwarte Bonn, 1904 Dez. I1. $9^{\mathrm{m}} \cdot 5 \quad 18^{\mathrm{h}} 40^{\mathrm{m}} 38^{\mathrm{s}}:+62^{\circ} 33^{\prime} \cdot 2$

der wohl mit dem Var. identisch sein könnte.

Der Stern var. 187.1904 Persei $=$ BD. $+39^{\circ} 79799^{\mathrm{m}_{5}}$ $3^{\mathrm{h}} 21^{\mathrm{m}} 0^{5} \cdot 7+39^{\circ} 9: 4$ ist in folgenden Sucherzonen beobachtet:

Z. I040 Sch. I 856 Sept. 30 , etwas dunstig $9^{\mathrm{m}} \cdot 5 \quad 3^{\mathrm{h}} 21^{\mathrm{m}} 0^{\mathrm{s}}: 0+39^{\circ} 7: 5$

Z. I 55 Sch. I 857 Febr. I4, sehr klare Luft $9^{\mathrm{m}} \cdot 5 \quad 3^{\mathrm{h}} 21^{\mathrm{m}} 1^{\mathrm{s}} \cdot 3+39^{\circ} 11: 2$

Ferner ist utber die Stelle gegangen:

Z. ro99 Sch. I 856 Okt. 29, meist klare Luft der Stern fehlt hier am Rande der Zone. 